\title{
SOME MATRIX TRANSFORMATIONS AND ALMOST CONVERGENCE
}

\author{
Neyaz Ahmad Sheikh, Ab. Hamid Ganie \\ Department of Mathematics, National Institute of Technology Srinagar - 190006. \\ Corresponding address: neyaznit@yahoo.co.in \\ Received 16 May, 2012; Revised 9 August, 2012
}

\begin{abstract}
The sequence space $b v(u, p)$ has been defined and the classes $\left(b v(u, p): l_{\infty}\right),(b v(u, p): c)$ and $\left(b v(u, p): c_{0}\right)$ of infinite matrices have been characterized by Başar, Altay and Mursaleen ( see, [2] ). The main purposes of the present paper is to characterize the classes $\left(b v(u, p): f_{\infty}\right),(b v(u, p): f)$ and $\left(b v(u, p): f_{0}\right)$, where $f_{\infty}, f$ and $f_{0}$ denotes the spaces of almost bounded sequences, almost convergent sequences and almost convergent null sequences, respectively, with real or complex terms.
\end{abstract}

2010 AMS Mathematical Subject Classification: 46A45; 46B45; 40C05.

Keywords and Phrases: Sequence space of non-absolute type, almost bounded sequences, $\beta$-duals and Matrix mappings.

\section{INTRODUCTION, BACKGROUND AND PRELIMINARIES}

A sequence space is defined to be a linear space with real or complex sequences. Throughout the paper $\mathbb{N}, \mathbb{R}$ and $\mathbb{C}$ denotes the set of non-negative integers, the set of real numbers and the set of complex numbers, respectively. Let $\ell_{\infty}, c$ and $c_{0}$ respectively be Banach spaces of bounded, convergent and null sequences $x=\left\{x_{n}\right\}_{n=0}^{\infty}$ normed by $\|x\|=\sup _{n \geq 0}|x(n)|$; also, by $c s$ we denote the sequence of all convergent series(see, [7]).

Let $X$ and $Y$ be two non-empty subsets of the space $\omega$ of real or complex sequences. Let $A=\left(a_{n k}\right),(n, k \in \mathbb{N})$, be an infinite matrix of real or complex numbers. We write $(A x)_{n}=$ $A_{n}(x)=\sum_{k} a_{n k} x_{k}$. Then $A x=\left\{A_{n}(x)\right\}$ is called the $A$-transform of $x$, whenever $A_{n}(x)=$ $\sum_{k} a_{n k} x_{k}$ converges for each $n \in \mathbb{N}$. We write $\lim _{n} A x=\lim _{n} A_{n}(x)$. If $x \in X$ implies $A x \in Y$, we say that $A$ defines a (matrix) transformation from $X$ into $Y$ and we denote it by $A: X \rightarrow Y$. By $(X: Y)$, we mean the class of all matrices $A$ such that $A: X \rightarrow Y$.

Let $D$ denote the shift operator on $\omega$, that is , $D x=\{x(n)\}_{n=1}^{\infty}, D^{2} x=\{x(n)\}_{n=2}^{\infty}$ and so on. Obviously, $D$ is a bounded linear operator on $l_{\infty}$ onto itself. A Banach limit $L$ is a non-negative linear functional on $l_{\infty}$ such that $L$ is invariant under the shift operator that is, $L(S x)=L(x)$ and that $L(e)=1$, where $e=\{1,1, \ldots\}$ (see, [1]). A sequence space is said to be almost convergent (see, [3]) to the generalized limit $\alpha$ if all Banach limits of $x$ are $\alpha$. We denote the set of almost convergent sequences by $f$. It was proved by Lorentz (see, [3]) that

$f=\left\{x \in l_{\infty}: \lim _{m} \tau_{m n}(x)=\alpha\right.$, uniformly in $\left.n\right\}$,
where, $\quad \tau_{m n}(x)=\frac{1}{m+1} \sum_{j=0}^{m} x_{j+n}, \tau_{-1, n}=0$ and $\alpha=f$-lim $x$.

Nanda [6] has defined a new set of sequences $f_{\infty}$ as follows:

$$
f_{\infty}=\left\{x \in l_{\infty}: \lim _{m}\left|\tau_{m n}(x)\right|<\infty\right\} .
$$


We call $f_{\infty}$ the set of all almost bounded sequences.

We denote by $X^{\beta}$, the $\beta$-dual of a sequence space $X$ and mean the set of all these sequences $x=\left(x_{k}\right)$ such that $x y=\left(x_{k} y_{k}\right) \in c s$ for all $y=\left(y_{k}\right) \in X$.

The approach of constructing a new sequence space by means of matrix domain of a particular limitation method has been studied by several authors viz., ( $[2,4,5])$.

The sequence space $b v(u, p)$ has been defined and the various classes $\left(b v(u, p): l_{\infty}\right)$ $(b v(u, p): c)$ and $\left(b v(u, p): c_{0}\right)$ have been characterized (see, [2]). In the present paper, we characterize the classes $\left(b v(u, p): f_{\infty}\right),(b v(u, p): f)$ and $\left(b v(u, p): f_{0}\right)$, where $u=\left(u_{k}\right)$ is a sequence such that $u_{k} \neq 0$ for all $k \in \mathbb{N}$.

The space $b v(u, p)$ is defined (see, [2]) as

$$
b v(u, p)=\left\{x=\left(x_{k}\right) \in \omega: \sum_{k}\left|u_{k} \Delta x_{k}\right|^{p_{k}}<\infty\right\},
$$

where, $\Delta x_{k}=x_{k}-\Delta x_{k-1}$.

\section{MAIN RESULTS}

Define the sequence $y=\left(y_{k}\right)$ which will be used as the $A^{u}$-transform of a sequence $x=$ $\left(x_{k}\right)$, i.e.,

$$
y_{k}=u_{k} \Delta x_{k} ; k \in \mathbb{N}
$$

For brevity in notation, we write

$$
\begin{array}{ll} 
& t_{m n}(x)=\frac{1}{m+1} \sum_{j=0}^{m} A_{n+j}(x)=\sum_{k} a(n, k, m) x_{k}, \\
\text { where, } & a(n, k, m)=\frac{1}{m+1} \sum_{j=0}^{m} a_{n+j, k} ;(n, k, m \in \mathbb{N}) \\
\text { Also, } & \bar{a}(n, k, m)=\left[\frac{a(n, k, m)}{u_{k}}\right] ;(n, k, m \in \mathbb{N}) .
\end{array}
$$

Now, we give the following lemmas which will be needed in proving the main Theorems.

Lemma 2.1 [2] : Define the sets $D_{1}(p)$ and $D_{2}(p)$ as follows:

$$
\begin{array}{ll}
D_{1}(p)=\left\{a=\left(a_{k}\right) \in \omega: \sup _{n} \sum_{k}\left|\sum_{j=k}^{n} \frac{a_{j}}{u_{k}}\right|^{p_{k}}<\infty\right\}, \\
& D_{2}(p)=\bigcup_{B>1}\left\{a=\left(a_{k}\right) \in \omega: \sup _{n} \sum_{k=0}^{n}\left|\sum_{j=k}^{n} \frac{a_{j}}{u_{k}} B^{-1}\right|^{p_{k}^{\prime}}<\infty\right\} . \\
\text { Then, } & {[b v(u, p)]^{\beta}=D_{1}(p) \cap c s ;\left(0<p_{k} \leq 1\right)} \\
\text { and } & {[b v(u, p)]^{\beta}=D_{2}(p) \cap c s ;\left(1<p_{k}<\infty\right) .}
\end{array}
$$

Lemma 2.2 [6]: $f \subset f_{\infty}$.

We consider only the case $1<p_{k} \leq M<\infty$ and the case $0<p_{k} \leq 1$ may be proved in a similar fashion.

Theorem 2.3: (a) Let $1<p_{k} \leq M<\infty$ for every $k \in \mathbb{N}$. Then $A \in\left(b v(u, p)\right.$ : $\left.f_{\infty}\right)$ if and only if

and

$$
\begin{aligned}
& \sup _{n, m} \sum_{k}\left|\bar{a}(n, k, m) B^{-1}\right|^{p_{k}^{\prime}}<\infty \\
& \left\{a_{n k}\right\} \in D_{2}(p) \cap c s .
\end{aligned}
$$


(b) Let $0<p_{k} \leq 1$ for every $k \in \mathbb{N}$. Then $A \in\left(b v(u, p)\right.$ : $\left.f_{\infty}\right)$ if and only if $\sup _{n, m} \sum_{k}|\bar{a}(n, k, m)|^{p_{k}}<\infty$

and

$$
\left\{a_{n k}\right\} \in D_{1}(p) \cap c s \text {. }
$$

Proof : Sufficiency: Suppose the conditions holds and $x \in b v(u, p)$.Using the inequality which holds for any $C>0$ and any two complex numbers $a, b$

$$
|a b| \leq C\left\{\left|a C^{-1}\right|^{q}+b^{p}\right\}
$$

where, $p>1$ and $p^{-1}+q^{-1}=1$ (see, [3]), we have

$$
\begin{aligned}
\left|t_{m n}(A x)\right| & =\left|\sum_{k} a(n, k, m) x_{k}\right|=\left|\sum_{k} \bar{a}(n, k, m) y_{k}\right| \\
& \leq \sum_{k} B\left[\left|\bar{a}(n, k, m) B^{-1}\right|^{p_{k}^{\prime}}+\left|y_{k}\right|^{p_{k}}\right]
\end{aligned}
$$

Now, taking sup over $m, n$ on both sides to the above inequality, we get $A x \in f_{\infty}$ for every $x \in b v(u, p)$, i.e., $A \in\left(b v(u, p): f_{\infty}\right)$.

Necessity: Suppose that $A \in\left(b v(u, p): f_{\infty}\right)$. Then $A x$ exists for every $x \in b v(u, p)$, and this implies that $\left\{a_{n, k}\right\}_{k \in \mathbb{N}} \in[b v(u, p)]^{\beta}$ for every $n \in \mathbb{N}$, the necessity of (2.3) is immediate.

Now, $\sum_{k} a(n, k, m) x_{k}$ exists for each $m, n$ and $x \in b v(u, p)$, the sequences $\{a(n, k, m)\}_{k \in \mathbb{N}}$ define the continuous linear functionals $\varphi_{m n}(x)$ on $b v(u, p)$ by $\varphi_{m n}(x)=\sum_{k} a(n, k, m) x_{k}$; $n, k, m \in \mathbb{N}$. Since $b v(u, p)$ is complete and $\sup _{m, n}\left|\sum_{k} \bar{a}(n, k, m) x_{k}\right|<\infty$, so by uniform bounded principle, there exists $M>0$ such that

$$
\begin{aligned}
\sup _{m, n}\left|\varphi_{m n}(x)\right| & =\sup _{m, n}\left|\sum_{k} a(n, k, m) x_{k}\right| \\
& =\sup _{m, n}\left|\sum_{k} \bar{a}(n, k, m) x_{k}\right| \leq M<\infty
\end{aligned}
$$

This implies that $\sup _{m, n} \sum_{k}\left|\bar{a}(n, k, m) x_{k}\right|^{p_{k}^{\prime}}<\infty$, which shows the necessity of the condition (2.2) and the proof of (i) is complete.

Theorem 2.4 : (a) Let $1<p_{k} \leq M<\infty$ for every $k \in \mathbb{N}$. Then $A \in\left(b v(u, p): f_{\infty}\right)$ if and only if (i) the condition (2.2)-(2.5) of Theorem 2.3 holds

(ii) there is a sequence $\left(\beta_{k}\right)$ of scalars such that $\lim _{m} \bar{a}(n, k, m)=\beta_{k}$, uniformly in $n$.

Proof: Sufficiency: Suppose that the conditions (2.2)-(2.6) hold and $x \in b v(u, p)$. Then $A x$ exists and we have by (2.6) that $\left|\bar{a}(n, k, m) B^{-1}\right|^{p_{k}^{\prime}} \rightarrow\left|\beta_{k} B^{-1}\right|^{p_{k}^{\prime}}$ as $m \rightarrow \infty$ uniformly in $n$ for each $k \in \mathbb{N}$, which leads us with (2.2) that

$$
\begin{aligned}
\sum_{j=0}^{k}\left|\beta_{j} B^{-1}\right|^{p_{k}^{\prime}} & =\sum_{j=0}^{k}\left|\bar{a}(n, j, m) B^{-1}\right|^{p_{k}^{\prime}} \\
& \leq \sup _{m, n} \sum_{j}\left|\bar{a}(n, j, m) B^{-1}\right|^{p_{k}^{\prime}}<\infty,
\end{aligned}
$$

holding for every $k \in \mathbb{N}$. Consequently reasoning as in the proof of the sufficiency of Theorem 2.3 , the series $\sum_{k} a(n, k, m) x_{k}$ and $\sum_{k} \beta_{k} x_{k}$ converges for every $n, m$ and for every $x \in$ $b v(u, p)$. Now, for given $\varepsilon>0$ and $x \in b v(u, p)$, choose a fixed $k_{0} \in \mathbb{N}$ such that 
$\left[\sum_{k=k_{0}+1}^{\infty}\left|x_{k}\right|^{p_{k}}\right]^{\frac{1}{H}}<\varepsilon$, where $H=\sup _{k} p_{k}$. Then, there is some $m_{0} \in \mathbb{N}$, by condition (ii) such that $\left|\sum_{k=1}^{k_{0}}\left[a(n, k, m)-\beta_{k}\right]\right|<\varepsilon$, for every $m \geq m_{0}$ and uniformly in $n$.

Now, since $\sum_{k} a(n, k, m) x_{k}$ and $\sum_{k} \beta_{k} x_{k}$ converges (absolutely) uniformly in $n, m$ and for $x \in b v(u, p)$, we have that $\sum_{k_{0}+1}^{\infty}\left[a(n, k, m)-\beta_{k}\right] x_{k}<\frac{\varepsilon}{2}$, converges uniformly in $n, m$ and $x \in b v(u, p)$. Hence by conditions (i) and (ii) we have $\sum_{k_{0}+1}^{\infty}\left[a(n, k, m)-\beta_{k}\right]<\frac{\varepsilon}{2}$ for all $\left(m \geq m_{0}\right)$, uniformly in $n$. Therefore, $\left|\sum_{k_{0}+1}^{\infty}\left[a(n, k, m)-\beta_{k}\right]\right| \rightarrow 0(m \rightarrow \infty)$ uniformly in i.e.,

$$
\lim _{m} \sum_{k} a(n, k, m) x_{k}=\sum_{k} \beta_{k} x_{k} \text { uniformly in } n .
$$

Hence, $A x \in f$, which proves sufficiency.

Necessity: Suppose that $A \in(b v(u, p): f)$. Then, since $f \subset f_{\infty}$ ( by Lemma 2.1 ), the necessities of condition (i) is immediately obtained from Theorem 2.1 . To prove the necessity of (ii) i.e., (2.6), consider the sequence $e_{k}=\left(0,0, \ldots, 1^{k t h-p l a c e}, 0,0, \ldots\right) \in b v(u, p)$, condition (ii) follows immediately by (2.7) and the proof is complete.

Collary 2.5: $A \in\left(b v(u, p): f_{0}\right)$ if and only if condition (i) and (ii) of above Theorem holds along with $\beta_{k}=0$ for each $k \in \mathbb{N}$.

Proof: The proof follows from theorem 2.4 by taking $\beta_{k}=0$ for each $k \in \mathbb{N}$.

\section{REFERENCES}

[1] Banach, S, Theòries des operations linéaries, Warszawa, 1932.

[2] Başar, F, Altay, B \& Mursaleen, M , Some generalizations of the space $b_{p}$ of p-bounded variation sequences, Nonlinear Anal., 68 (2)(2008), 273-287.

[3] Lorentz, G G, A contribution to the theory of divergent series, Acta Math., (80) (1948), 167-190.

[4] Mursaleen, M, Infinite matrices and almost convergent sequences, Southeast Asian Bulletin of Math., 19(1) (1995), 45-48.

[5] Mursaleen, M, Jarrah, A. M. \& Mohiuddine, S. A., Almost convergence through the generalized de la Vallee-Pousin mean, Iranian J. Sci. Tech. Trans. A, 33(A2) (2009), 169-177.

[6] Nanda, S, Matrix transformations and almost boundedness, Glasnik Mat., 14(34) (1979), 99-107.

[7] Yasida, K, Functional Analysis, Springer-Verlag, Berlin Heidelberg, New York, 1966. 